\title{
Methods of Processing Context in Intelligent Systems
}

\author{
Iryna Zavuschak \\ PhD-student of the Department of Information Systems and Networks, \\ Lviv Polytechnic National University \\ Lviv, 79013, Ukraine \\ Email: iryska2009@ukr.net
}

Received: 27 December 2017; Accepted: 30 January 2018; Published: 08 March 2018

\begin{abstract}
Context-based methods represent an important part of toolkit used to build intelligent systems. In the article existent definitions of context for a system with a single decision making agent were discussed. Available formal models for context data representation and processing were compared. The approaches for different forms of reasoning within context were analyzed. Also the application of context awareness in systems with situation awareness is discussed. In the article unresolved problems and tasks in the domain of context aware computing are delineated.
\end{abstract}

Index Terms - Data, knowledge, context, database, knowledge base.

\section{INTRODUCTION}

Possession of knowledge makes it possible to develop and achieve success in solving problems of intelligent systems. On the basis of existing knowledge, in the process of analytical thinking, new findings appear, thereby gaining new knowledge. The process of creating knowledge can be called infinite, and an important role in this process is dedicated to the accumulation and transfer of knowledge gained.

In solving problems and information exchange between systems, not all data is clearly defined - a significant number can be provided indirectly. This, nondirect way, can be implemented through:

- Determination of the relevant environment for solving the problem. Such characteristics may be time, place, and other circumstances.

- Implicit assumptions and limitations imposed by system designers.

- Previous experience of participants. Some facts used in the process of solving problems can have a radically different interpretation, in case participants have some specific experience.

- Preliminary stages of the problem solving process. For example, the use of pronouns in the language is almost entirely based on this principle.
Thus, solving problems in the intellectual system requires the correct understanding and use of implicit data, information and knowledge [1]. The absence of explicit definition and processing of the context is considered one of the reasons for failures in the construction of intelligent systems [2]. The task of creating context aware systems is of particular importance in today's environment through the development and diffusion of autonomous intelligent systems.

The purpose of this work is to analyze the existing methods and means of conveying and processing the context and defining the directions of their development, taking into account the tasks of constructing intelligent decision support systems.

Given the close relationship between context-sensitive systems and knowledge-based systems, the concept of "context", the types and properties of the context will first be considered. The next part of the paper will be devoted to the analysis of formal methods for representing and working out the context. In the third part we will consider and analyze the application of reasoning (reasoning - the result of reflection, views, persuasion) and logical output for context-based computing. In the last part we consider the connection of the processing of the context with the task of forming situational awareness.

\section{RELATED WORKS}

To date, the notion of context is used in linguistics, robotics, intellectual and mobile systems. At the same time, there is no common definition for the context for these industries [3]. Separate studies examine partial cases of the context or even their individual properties. This leads to a discrepancy in the interpretation and use of context-sensitive methods, the impossibility of reusing methods and models created for different industries and even for solving problems in one industry. Therefore, it is advisable to analyze existing definitions and methods for using context data in order to highlight their common features and properties. This, in turn, enables the same interdisciplinary interpretation of the concept of context 
and the creation of on its basis methods and tools that reuse contextual knowledge.

Encyclopedic and vocabulary sources give the following definition of the term "context":

- The context is "interconnected conditions in which something exists or occurs" [4]

- $\quad$ "A situation in which something exists or occurs and which can be used to explain this" [5]

- "A set of conditions or facts that determine a particular event or situation" [6]

- Conditions that form the environment for an event, sentence or idea, and provide timelines for their understanding" [7]

- In [8], the context is defined as "arbitrary information that can be used to characterize a particular entity."

Thus, the general understanding of the concept of "context" involves the presence of a central Agent object (which can also be a multi-agent system or object) and the outside world (World). The part of the external world used to understand the object or to decide on the agent is Context (Fig. 1). The context contains the object itself. The content of the context is determined by the characteristics of the object, as well as the goals and limitations specified for the agent. Therefore, the contextsensitive SysContext system is a triple:

\section{SysContext $=($ Agent, World, Context $)$}

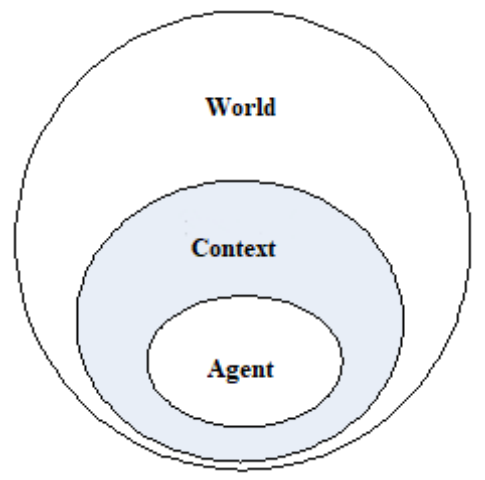

Fig.1. Object, context and the outside world

Context reduces the space in which searches are sought for information when solving problems with incomplete or incorrect inputs. Therefore, an important practical point of view is the solution to the problem of determining the minimum amount of context information that is necessary to solve the problems faced by the agent.

\section{WORKING KNOWLEDGE IN THE CONTEXT - DEPENDENT SYSTEMS, TYPES AND PROPERTIES OF CONTEXT}

In practice, the context-sensitive system perceives the world indirectly by obtaining and analyzing information from many sources. Such sources, for example, include sensors, databases and knowledge, memory. The perception and processing of context data takes place within the limits and means of the intellectual system, using existing knowledge of the laws and dependencies in the subject area, the previous stages of the system [9].

Knowledge, in particular, is used for the semantic interpretation of sensor data, the processing of information thus obtained and the formation of new information and knowledge.

On the other hand, knowledge is often contextdependent, that is, true only in a certain context. Therefore, it is not surprising that in powerful expert systems such as CYC [10], logical rules are true or false only for a certain defined context. An important problem for intelligent decision support systems is to determine the relevance of knowledge - that is, the search for knowledge that is appropriate to use in an existing context.

In [11] distinguish knowledge about the order of the task (know how) and knowledge of the subject area (know that), deep knowledge and surface knowledge (deep and surface knowledge), procedural and declarative knowledge, explicit and implicit knowledge. In accordance with these forms of knowledge is defined [11]

- Proceduralized context (context) as a form of contextual knowledge that is needed to solve a specific task;

- Context knowledge as implicit knowledge about the features of the subject area.

The variability of knowledge about the environment is reflected in the concepts of static and dynamic context. In particular,

- The static context contains knowledge that does not change during object interaction. They correspond to the knowledge of the subject area.

- The dynamic context describes an evolving part of knowledge. The dynamic nature of the context is manifested when it is used by object objects, for example, when solving a problem [12].

Definitions and context properties given in [1, 2, 13] consider the context that arises as a result of the interaction of objects. However, this definition does not answer the question of what is the choice of interacting objects, why and how the context changes over time. In an approach that considers the context of the agent, adopted in this article, the key is the agent context, its properties, and the task it faces. In fact, they determine the rational behavior of the object in a certain environment. The choice of environment elements in the context is determined by their importance and influence on the task solved by the agent [14]. The main properties of the context are given in Table. 1 
Table 1. Properties of the context

\begin{tabular}{|c|c|c|}
\hline № & Property & Consequence \\
\hline 1 & The context is determined by the agent & $\begin{array}{l}\text { The context contains the agent itself and part of the environment that is } \\
\text { relevant to the set of tasks that the agent solves. }\end{array}$ \\
\hline 2 & $\begin{array}{l}\text { The general context that occurs when an agent solves tasks } \\
\text { involves objects contexts that are relevant to solving problems }\end{array}$ & $\begin{array}{l}\text { Considering agent contexts and directly relevant objects allows you to } \\
\text { limit the number of objects that processes the system. At the same time, } \\
\text { if we consider the contexts of objects associated with the relevant } \\
\text { objects, then the dimension of the problem may increase. }\end{array}$ \\
\hline 3 & $\begin{array}{l}\text { The context is boundless. It is impossible to fully determine it } \\
\text { [12] }\end{array}$ & $\begin{array}{l}\text { Given the context of all indirectly dependent objects, the context } \\
\text { expands to cover the whole world. At the same time, the complexity of } \\
\text { processing such a context increases accordingly. Thus, the important } \\
\text { task is to constrain the size of the context to the minimum necessary for } \\
\text { solving the tasks, and the development of effective methods of } \\
\text { contextual search. }\end{array}$ \\
\hline 4 & $\begin{array}{l}\text { If the context of the system is considered from several agents, } \\
\text { then there is a common context for them }\end{array}$ & $\begin{array}{l}\text { It is appropriate to consider this common context taking into account the } \\
\text { goals of different interacting objects, that is, from different perspectives } \\
\text { that may differ }\end{array}$ \\
\hline 5 & $\begin{array}{l}\text { The context in the process of solving tasks by the agent may } \\
\text { vary }\end{array}$ & As a result of such changes, objects change their relevancy. \\
\hline 6 & The context includes implicit knowledge and information & $\begin{array}{l}\text { This property has two consequences: } \\
\text { 1) as much contextual information as possible should be presented } \\
\text { explicitly; } \\
\text { 2) using the history of receiving and changing the context allows you to } \\
\text { display a new context based on previously used contexts. }\end{array}$ \\
\hline 7 & $\begin{array}{l}\text { The context can be described as the relationship between } \\
\text { elements of abstract models, as well as specific instances of } \\
\text { abstraction data }\end{array}$ & $\begin{array}{l}\text { This property has as a consequence the ability to create an abstract } \\
\text { context from the current context used, which can be further specified for } \\
\text { other conditions. The process of creating an abstract context is called } \\
\text { decontextualization. }\end{array}$ \\
\hline
\end{tabular}

Many of the existing context-sensitive systems focus on the processing of the procedural context, while the task is to effectively use deep, context-sensitive knowledge for reasoning and logical output remains unresolved.

\section{A Formal methods of representation and processing of context}

The choice of the formal method for representing and processing the context is largely determined by the set of tasks that the intellectual system solves and the ways in which knowledge is presented and developed in it. Historically, at the early stages of the development of context-dependent systems, approaches based on the predicate logic and production rules prevailed. At present, the most popular method of presenting and processing the context are methods of ontological engineering.

The paper [15] sets out the main requirements for formal models of presentation and processing of the context. Such requirements are:

- Applicability - the ability to apply a context model to a specific subject area and task.

- The possibility of a correct comparison of data. Sensors that provide contextual information often have different coding schemes, possibly a different interpretation of these data.

- Tracking changes. For the correct interpretation of context data, you need to know the origin of all data and all changes made to them

- Determining the quality of information and the possible level of errors obtained from different sources of information, the level of trust in sources.

- Possibility of logical deduction and other beliefs

Key-value models provide contextual information for the interpretation of certain variables in the form of environment variables. For example, such variables may include running location, user ID, formatting information on a page, etc. Key-value models are widely used in software systems, they are easy to use and configured, but not structured, which makes it impossible to build efficient context management systems [16].

Markup languages use hierarchical markup tag structure with their attributes and content to represent contextual information. In this case, the content of the tag is often determined recursively by other tags. Documents specifying the use of this class of contextual models are diverse profiles, such as Composite Capabilities / Preferences Profile (CC / PP), Comprehensive Structured Context Profiles (CSCP), Pervasive Profile Description Language (PPDL), and others. They define the hierarchy of the component-attribute pairs for specific areas of use. Markup languages and profiles allow you to identify context elements for a specific set of tasks, but they do not reflect the patterns and dependencies that exist in the subject area.

The most well-known representative of graphical and object-oriented forms of context representation is $U M L$ (Unified Modeling Language), and its Object Role Modeling (ORM) context extension. ORM and its extension are used to represent the facts of the domain and the facts dependent on them. UML and ORM are used to design database structures and document 
dependencies in a subject domain. At the same time, they are not suitable for constructing complex models and algorithms for the processing of contextual information, but do not support the hierarchical structure of facts.

Context Logic - Applies to a context that is defined as a set of axioms. This logic is an extension of the logic of the first order, where the axioms are considered to be faithful or untrue within a certain context. The context is given by the formal object and the relation ist $(c, p)$, where $\mathrm{c}$ is the context, $\mathrm{p}$ is an axiom.

The relation ist $(c, p)$ declares that the axiom $\mathrm{p}$ is true in the context of c. Contextual management is carried out using axioms that transfer from one context to another. The transposed axiom in the new context should have the same conditions of truth that it had in the original context [13].

Propositional statements - the aforementioned axioms, expressed in sentences, are used to formalize contexts in the multicontextual knowledge base of Cyc [17]. According to the relation ist $(c, p)$, the context is defined as an agreed set of propositional statements. The scope of the context is determined by the set of objects, conditions, etc., for which the statements about them are correct. Between contexts are hierarchical relationships that allow the formation of contexts of different depth of detail. Context management is done through procedures that perform the logical conclusion of expressions, described in general contexts, in specialized contexts.

The rules system is used for contexts describing the structure of knowledge. The formalism of the system of rules gives the structure of knowledge to the rules of the packets. The representation is at the level of rules and at the knowledge base level. The rule-level submission is controlled by filtration expressions.

Knowledge-level presentation divides the knowledge base into sets of individual small databases that are directly controlled by the rules that cause the rule packets in the "then" part, or iterations between the rule packets for the exchange of information. The disadvantages of systems based on the rules are the lack of a holistic model of the domain, the fragmentation of the rules packets and the complexity of their alignment and maintenance.

The most used formal means of presenting the context today is ontology. Ontology, as a formal conceptualization of a particular subject area, enables one to define the concepts of this area and the relation between them. This definition enables a common understanding and sharing of knowledge by various context-based applications [18]. Contrary to the ontology of the subject area, context-based ontology defines the objects and relationships that are relevant to the problem solved by the agent at a given time [13].

From the point of view of the way in which the context is presented, it should be described in standardized ways that ensure the independence of the presentation from the platform. The knowledge representation model should support operations for creating and managing the context. Contextual ontology includes:
1. Concepts and their properties, representing objects involved in the situation in which the agent is located;

2. Relevant to the situation, the relation that connects these concepts and their properties;

3. Fundamental constraints on the situation.

Context Ontology is part of the general ontology. Restricting the size of the context ontology simplifies understanding and decision making in the context.

The content and structure of the context ontology change in the process of functioning of the intellectual agent because the changing environment and running tasks that the agent solves. For such dynamic contexts, at each step, one has to solve the task of constructing a context ontology. In this case, both general ontology of the subject domain and other ontologies are often used. In [13], the following methods and formalisms for dynamic determination of context ontology are analyzed:

- Algebra of ontologies [19]. Contextual ontology is based on several initial ontologies. The elaborate algebra supports selections, combinations, intersections and determinations of ontology differences.

- Combining ontologies Contextual ontology is obtained as a result of combining parts of different ontologies that are relevant to the situations in which the agent is located. As a result of this association, an inconsistent ontology is obtained.

- Display of ontologies. This approach is used to integrate heterogeneous sources of information and knowledge and the main ontology, in particular in the Cyc system [17].

In [9], context-based ontology is constructed on the basis of the running state of the agent as the central object of the situation on the basis of existing factsrelations.

In addition to constructing a context ontology for context-sensitive decision-making tasks, it is important to identify and formalize contextual dependencies in the formal model. Actually contextual dependencies, presented by a chain of related facts, make it possible to determine the meaning of a particular parameter in the context that is used to make a decision. The method of representing and using contextual dependencies as chain of concepts and facts is proposed in [9].

A number of ontologies have been developed to solve context-dependent computer problems. The most famous of them are SOUPA, CONON, ConOnto, ConOL, mySAM, $A S C$ and others. The paper [15] defines the main criteria for comparing ontologies, which to a large extent relate to the quality of ontology in general, and not only to address contextual tasks. Such criteria are standardization, flexibility and the ability to expand, universality, granularity, integrity, completeness, lack of redundancy, scalability, and the use of formal languages. 
Thus, in the field of formal methods of presentation and processing of the context, the tasks of developing effective methods of dynamic determination of relevant contextual ontologies, analysis of contextual dependencies, taking into account the fuzzy and unreliable context information remain unresolved.

\section{B. Mathematical model presentation of knowledge}

The use of ontological models for the construction of software systems requires the development of mathematical justification in the form of a corresponding formal model, which will be further used for the formal presentation and validation of methods and modeling systems. To construct a formal model approach used algebra systems [5], this determines the algebraic system by combining several algebraic domains.

Let in the subject region there are many objects:

$$
A_{1}, A_{2}, \ldots, A_{n}
$$

Objects belonging to each plural are classified as instances of a particular concept. These sets are multiplicative carriers for much multi-type algebra. Individual instances that belong to this for sets, denote $\mathrm{a}_{1}, \ldots, \mathrm{a}_{\mathrm{n}}$.

Domains of Concepts (Essences) E. On the basis of each set $A_{i}$ we define the abstract data type $E_{i}$

$$
E_{i}=\left(\text { Name }, \sum, E x\right),
$$

where, Name is the type name; $\sum$ - signature of a multisorted algebra; $E x$ - determinants of type. In this case, the signature contains only a plurality of elements and does not contain operations and relationships.

Separate types will denote $E_{i}$ (an arbitrary type, its name is not defined) or $E_{\text {name }}$ where name is the type name if it is important to specify a specific type in the context of the consideration. Variables that acquire the values of instances of a particular type are denoted by $X_{\text {name }}$, or simply Name. Types $E_{i}$ form the set of algebraic domains $E$, which correspond to the concepts (entities) of the subject domain.

$$
E=\left\{E_{1}, E_{2}, \ldots, E_{n}\right\}
$$

Attributes Domain At. Define the algebraic attribute domain $A t$ at the list of attribute values in pairs (key, value). The key element of the key determines the attribute ID, and value is its value. According to [5], this merger, substitution, deletion, interpretation (merge, substitute, delete, interp) operations are defined for this domain.

In practice, functions that are defined on variables of different domains are useful, for example, the function of choosing an attribute value:

$$
F_{\text {selval }}(\text { At, key }) \rightarrow \text { value. }
$$

Boolean domain Cs. The Boolean domain includes expressions whose counting results belong to the Boolean set \{true, false\}. Operands of expressions are variables belonging to other algebraic domains. We will interpret boolean domain elements as Cs constraints. Operations for a boolean domain are boolean operations \{and, or, negation \}, as well as an interp interpretation operation that displays simplicity and counting boolean expressions. An instance of a boolean domain is a specific restriction.

Domain entities with attributes $\mathbf{T}$. Defined on the set of tuples $\left\langle E_{i}, A t_{j}, C s_{j}{ }^{*}\right\rangle$. For each $i$ there is only one $j$ that is included in the element of this domain:

$$
\forall i \exists^{1} j:<E_{i}, A t_{j}>
$$

Each limitation $\mathrm{Cs}_{\mathrm{j}} \in \mathrm{Cs}_{\mathrm{j}}{ }^{*}$ is an expression with the operands belonging to the domain $\mathrm{At}_{\mathrm{j}}$. Operations over elements of this domain are merging and dividing entities \{merge, split\}. The operation of the entity of entities is interpreted as the formation of a new entity as a common set of properties and constraints of the essentialsoperands. The division of the entity is the reverse of the unification. An instance of an entity domain with attributes is a tuple of relevant facts.

Domain relations R1. The host number of this domain is the structure of the form:

$$
\left\{\left(T_{1, i} \times T_{2, i} \times \ldots \times T_{k, i}, A t_{i}^{r} C s_{i}^{*}\right)\right\} .
$$

Each structure is a tuple containing Cartesian product of algebraic data types from a domain $\mathrm{T}$, a type from the attribute domain At (defines attributes of the relation), and a set of restrictions Cs.

Each restriction $\mathrm{Cs}_{\mathrm{i}} \in \mathrm{Cs}_{\mathrm{i}}{ }^{*}$ is a Boolean expression with operands belonging to domains $\mathrm{At}_{1, \mathrm{i}}, \mathrm{At}_{2, \mathrm{i}}, \ldots, \mathrm{At}_{\mathrm{k}, \mathrm{i}}$, $\mathrm{At}_{\mathrm{i}}^{\mathrm{r}}$.

Above the relationships, the combining operations, separation, substitution \{merge, split, substitute are defined. Combining and separating transactions are treated similarly to operations of domain E. In the substitution operation, instead of one entity of the domain $\mathrm{T}$, we substitute the relation, which is interpreted as the essence with attributes (reification).

\section{The Use of Logical OutPut and REASONING IN The Process of Processing the ConteXt}

The use of knowledge in context-sensitive systems involves the use of formal domain models and logical output for the formation of new facts and knowledge based on contextual information. These facts, in turn, are used to support decision-making.

An important measure in the process of comparing different forms of reasoning is its depth, which is determined by the volume and complexity of information from the knowledge base that is used during reasoning. 
Thus, the processing of the procedural context is usually inadequate, because they take into account a small, preknown set of objects that are used to solve a particular task. In tasks that work with contextual knowledge, the degree of processing of information is deeper and the domain model is used.

In [20] the classification of different methods of reasoning is given depending on the volume and complexity of knowledge processing in them. These methods are summarized and supplemented in Table 2, which also highlights the advantages, disadvantages and scope of different methods for presenting and processing contextual data.

In particular, simple key-value models and markup languages such as XML or HTML use contextual data to add information about the code element. This information is used by browsers to display and verify data. Such simple models cannot be based on complex intellectual systems, because they are limited by rules and syntactic and semantic capabilities of a particular language.

Models based on product rules allow you to reflect more complex situations and apply in complex expert systems, for example, for medical diagnostics. The use of context data in them greatly complicates the system, since it requires the consideration of the correct set of rules within defined contexts. The disadvantage of systems based on rules is the lack of a coherent domain model, the complexity of matching rules and contexts.

Graphic models, including the ORM (Object Role Model), have been developed to display context dependencies when designing database schemas. They allow you to visually display contextual dependencies. The disadvantage of graph models is the "flat" nature of the model, the lack of support for hierarchy of concepts and inheritance of properties.

Ontology-based models are by far the most popular for presenting and processing contextual data. The ontology enables to formulate a formal hierarchical conceptual model of the domain and perform logical output operations based on the use of descriptive logic. Context is often presented as an ontology, or part of ontology [18] The disadvantages of using an ontological approach for contextual-dependent reasoning derive from a limited range of mental tasks supported by descriptive logic. These tasks include:

- Verification of belonging to a certain object;

- To imitate properties from other objects;

- Verification of the relation of consistency;

- Construction of new concepts using the axiom constructor.

Table 2. Forms of submission context for reasoning

\begin{tabular}{|c|l|l|l|l|}
\hline № & \multicolumn{1}{|c|}{ Form of submission } & \multicolumn{1}{|c|}{ Scope of use } & \multicolumn{1}{|c|}{ Advantages } & \multicolumn{1}{c|}{ Disadvantages } \\
\hline 1 & $\begin{array}{l}\text { Key-value models and } \\
\text { markup languages }\end{array}$ & $\begin{array}{l}\text { Verification of the } \\
\text { execution of simple } \\
\text { restrictions, verification }\end{array}$ & $\begin{array}{l}\text { Simplicity, do not require } \\
\text { significant computing resources }\end{array}$ & Limited range of tasks \\
\hline 2 & $\begin{array}{l}\text { Models based on product } \\
\text { rules }\end{array}$ & Expert systems & $\begin{array}{l}\text { Ability to use to solve complex } \\
\text { tasks }\end{array}$ & $\begin{array}{l}\text { Absence of an integrated domain } \\
\text { model. The difficulty of harmonizing } \\
\text { the rules and the contexts of their use }\end{array}$ \\
\hline 3 & Models based on graphs & $\begin{array}{l}\text { Modeling context } \\
\text { relationships between data }\end{array}$ & $\begin{array}{l}\text { Formation of the model of } \\
\text { contextual dependencies } \\
\text { between data }\end{array}$ & "Flat" character of the model. \\
\hline 5 & Using ontologies & $\begin{array}{l}\text { Intelligent decision support } \\
\text { systems }\end{array}$ & $\begin{array}{l}\text { Representation of the } \\
\text { hierarchical model of the } \\
\text { domain. Use of discretionary } \\
\text { logic for fragmentation }\end{array}$ & $\begin{array}{l}\text { It stems from the limitations of } \\
\text { descriptive logic }\end{array}$ \\
\hline Models deep reasoning & $\begin{array}{l}\text { Intelligent decision support } \\
\text { systems }\end{array}$ & $\begin{array}{l}\text { Getting new facts and } \\
\text { knowledge, deep knowledge of }\end{array}$ & $\begin{array}{l}\text { Significant needs in computing } \\
\text { resources. Bad scalability }\end{array}$ \\
\hline
\end{tabular}

Models of deep understanding combine various forms of reasoning, in particular, causal, probabilistic, or reasoning, based on modal, no monotonic or abductive logic.

One more direction of development of reason in the context is the implementation of multilevel understanding. In [18], the use of the primary context for the prior restriction of the search circle and the secondary context are considered for a deeper search in the set of documents defined in the previous step.

A common disadvantage of deep-thinking models when working out contextual data is the considerable cost of computing resources. Such models are poorly scaled with increasing number of facts that need to be included in the context of the task. An important task, therefore, is to reduce the size of the problem by predefining the concepts that are relevant to the problem of deep understanding.

\section{Use the Context in Situational Awareness SYSTEMS}

The concept of the context has much in common with the concept of the situation. Thus, the situation is determined in [19] "Facts, conditions and events affecting someone (at something) at a certain time and place." In the Ukrainian Academic Explanatory Dictionary, the notion of a situation is determined by the notion of position. Thus, the situation is a "set of conditions and circumstances that create a certain position, cause certain relations of people," and the 
situation - "Those or other circumstances, the conditions in which someone, something is" [15].

Scientific sources determine the situation as a set of dependent spatial and temporal facts. In classical works on the theory of situations, the terms "situation" and "context" are considered synonyms.

The task of using contextual-dependent knowledge for the formation of rational behavior of the intellectual agent requires the formation of his situational awareness. Situational awareness is based on the perception of the environment and the situation around them at the moment [18].

Situational awareness is a key element in decision support systems. In particular, in most cases, if the situation is correctly evaluated, it automatically determines the sequence of actions to be initiated. The methods that solve the problem of identification of problem situations allow formalizing knowledge of the expert about signs of problem situations accumulate and reuse the experience of decision-making in similar situations. This allows us to check the correctness of decisions in the historical perspective, comparing them with similar situations in the past. Correct identification of the situation involves solving the problem of detecting and taking into account contextual dependencies.

In turn, solving this problem requires in-depth study of existing and development of new principles and methods for formalizing knowledge of contexts in problem situations, models of their processing, creation of organizational, information and software related to decision-making support in context-sensitive systems.

An important task in the field of situational awareness is the study of the context at various stages of the JDL model of achieving situational awareness. Thus, in [19] it is suggested to use levels for determining the spatial context that are consistent with the general model of JDL, and in article [16] the framework of situational awareness for the problem of accident prevention. At the same time, the task of studying contextual dependencies for the general context of the context remains unresolved.

\section{CONCLUSIONS}

The general definition of the concept of context on the basis of the foregoing is that the context is described by an extremely large number of terms and is applicable in various fields. Having considered the definition of the context from different points of view, one can say that regardless of the field of application, the context is the data and knowledge relevant at a certain point in time and relevant to a particular task. In particular, the use of the Internet context explicitly increases the efficiency of web search. In the section of artificial intelligence, associated with the work of knowledge bases and ontologies, the context narrow down the areas of the database or knowledge bases, forming areas with significant information in a particular situation. Context is a tool for managing knowledge bases, which avoids redundancy and optimizes knowledge extraction. If we consider the context from the point of view of linguistics, in the language, as in interpersonal communication, the interpretation of a passage of text, language or expression depends on what was said (implied) before and after.

The orientation of the application to the use of the context allows, without affecting directly the logical conclusion, to restrict it only to rules or procedures that are meaningful for the given context. Context is a tool for managing knowledge bases, which avoids redundancy and optimizes knowledge extraction. Presenting a context in a formalized form allows the user to provide explanations about the program's actions, the chosen solution, the hypothesis, etc., based on the interpretation of the context. Thus, from the point of view of the application, the context allows more efficient use of the resources of the environment in which the system operates. From the perspective of the user, the context provides him with a valid, relevant, and accessible information for his task.

Despite the relevance of the field of context-sensitive computing, there are still many research areas and tasks that need to be addressed.

Thus, in the context of developing methods for representing and using the context, the development of methods is relevant, the advantage is the effective use of both the procedural context and contextual knowledge, depending on the particular situation. For dynamic systems it is important to trace the static and dynamic contextual contexts in order to reduce the dimensionality of the problem of its processing. The task of determining the relevance of contextual elements and developing methods for effectively presenting and finding elements in the context remains unresolved.

In the field of reasoning in the context, it is important to develop effective methods of deep-rooted thinking, including support for causative intelligence. At the same time it is necessary to solve the problem of a large resource-consuming problem of reasoning. One of the possible ways to achieve this goal is to develop methods for efficient use of the multi-level context.

Modeling contextual dependencies in situational awareness systems is a promising direction of contextsensitive computing. The task of studying contextual dependencies at all stages of the JDL model, definition of the peculiarities of their application is not solved.

The study of the context for incompletely defined, fuzzy, probabilistic data remains a promising direction, since such data are actually used in practice. Also relevant is the development of methods for using context data in support decision-making tasks for various subject areas, taking into account their structural features and solving tasks.

\section{REFERENCES}

[1] Bazire, M., July. Understanding context before using it./ Bazire, M., Brézillon P / Proceedings of International and Interdisciplinary Conference on Modeling and Using Context.- Springer Berlin Heidelberg, 2005.- P. 29-40.

[2] Brezillon P. Context in Artificial Intelligence: I. A Survey of the Literature / Brezillon P. // Computer \& Artificial Intelligence. - 1999. - № 4 - P. 321-340. 
[3] Melyara. Mezzi, Nadjia. Benblidia Study of Context Modelling Criteria in Information Retrieval - I.J. Information Technology and Computer Science, 2017, 3, 28-39 Published Online March 2017 in MECS (http://www.mecs-press.org/) DOI:10.5815/ijitcs.2017.03.04

[4] Context. Retrieved 1 Dec. 2016 from http://www.merriam-webster.com/dictionary/context

[5] Y. Burov. Formal model of knowledge in the system of ontological modeling of the problem /Ye. Burov // Proceedings of the National University "Lviv Polytechnic" series "Information systems and networks" Lviv, 2013 - p.21-30.

[6] Zavuschak I. The Context of Operations as the Basis for the Construction of Ontologies of Employment Processes /I. Zavuschak, Ye. Burov // Proceedings in a foreign edition «International Journal of Modern Education and Computer Science (IJMECS) - 2017. Vol.9. No.11. »Hong Kong: MECS Publisher - p.13-24, (http://www.mecs-press.org/)

[7] Salah Alkhafaji,B. Sriram Conceptualization and Integration of Information Systems in Educational Business Activities - I.J. Information Engineering and Electronic Business, 2013, 2, 28-33 Published Online August 2013 in MECS (http://www.mecs-press.org/) DOI: 10.5815/ijieeb.2013.02.05

[8] Y. Burov. Business process modelling using ontological task models./Burov Y//Econtechmod. - Lublin: Polish academy of sciences. - 2014. - №1. - P.11-23

[9] Burov Ye. Algebraic Framework for Knowledge Processing in Systems with Situational Awareness I (Khrystyna Mykich**) and Yevhen Burov // Advances in Intelligent Systems and Computing: Selected Papers from the International Conference on Computer Science and Information Technologies, CSIT 2016, September 6-10 Lviv, Ukraine".- Springer International Publisher, 2017.Vol.512.- P.217-228. - Access mode: http://www.springer.com/us/book/9783319459905.

[10] Lytvyn V. The process of managerial decision making support within the early warning system / V. Lytvyn, O.Tsmots. // Actual Problems of Economics. - № 11(149). - 2013. - SNIP 0,085.- P. 222-229. - (SCOPUS). Access mode: http://irbis-nbuv.gov.ua/cgibin/irbis_nbuv/cgiirbis_64.exe?C21COM=2\&I21...

[11] Burov E. Complex ontology management using task models /Burov E//International Journal of Knowledgebased and Intelligent Engineering Systems. - Amsterdam: IOS Press. - 2014. - Vol 18, no 2. - P. 111-120.

[12] Vysotska Victoria. Analysis Features of Information Resources Processing / Victoria Vysotska, Lyubomyr Chyrun // Computer Science and Information
Technologies: Proc. of the X-th Int. Conf. CSIT'2015, 1417 September, 2015, Lviv, Ukraine..- Lviv: Publishing Lviv Polytechnic, 2015.- P.124-128.

[13] Bazire, M., July. Understanding context before using it. / Bazire, M., Brézillon P / Proceedings of International and Interdisciplinary Conference on Modeling and Using Context.- Springer Berlin Heidelberg, 2005.- P. 29-40.

[14] Prem Nath Context-Sensitive Grammars and LinearBounded Automata - I. J. Computer Network and Information Security, 2016, 1, 61-66 Published Online January 2016 in MECS (http://www.mecs-press.org/) DOI: $10.5815 /$ ijcnis.2016.01.08

[15] Smirnov A.V. Models of Context-Managed Decision Support Systems in Dynamic Structured Regions / Smirnov A.V., Levashova T.V., Pashkin M.P. // Proceedings of SPIIRAN. - 2009. - №9. - P.116-147.

[16] Zavuschak I. Construction of the ontology of the processes of employment on the basis of analysis of the operations contexts / I. Zavuschak, E. Burov // Professional Journal of LNTU "Computer Integrated Technologies: Education, Science, Production". - Lutsk, 2017, issue number 27, p.105-119.

[17] I. Zavuschak, Z. Rybchak, I. Zheliznyak. Analysis of Clustering Algorithms - Advances in Soft Computing. Book Series of "Advances in Intelligent Systems and Computing”, ISSN 2194-5357. Springer International Publishing AG 2017. Germany

[18] Zavuschak I. The context analysis and the process of its formation /I. Zavuschak // Proceedings of the «ECONTECHMOD. An international quarterly journal 2017. Vol. 6. No. 2.» - Lublin : Polish Academy of Sciences Branch - p.67-72, ISSN: 2084-5715;

[19] Usha Yadav, Gagandeep Singh Narula, Neelam Duhan, Vishal Jain Ontology Engineering and Development Aspects: A Survey - I.J. Education and Management Engineering, 2016, 3, 9-19 Published Online May 2016 in MECS (http://www.mecs-press.net) DOI: 10.5815/ijeme.2016.03.02

\section{Authors' Profiles}

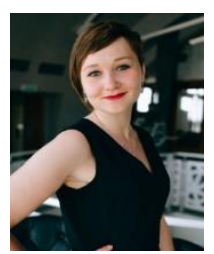

Iryna Zavuschak - a PhD-student, assistant of the Department of Information Systems and Networks, Institute of Computer Science and Information Technologies, of Lviv Polytechnic National University. Currently working on dissertation and engaged in scientific work.

How to cite this paper: Iryna Zavuschak, " Methods of Processing Context in Intelligent Systems", International Journal of Modern Education and Computer Science(IJMECS), Vol.10, No.3, pp. 1-8, 2018.DOI: 10.5815/ijmecs.2018.03.01 